\title{
Sliding over anisotropic beds
}

\author{
Righard G.A. Hindmarsh \\ British Antarctic Survey, Natural Environment Research Council, High Cross, Cambridge CB3 OET, England
}

\begin{abstract}
Many glacier beds are anisotropic, by which is meant that the dominant wavelengths are different in the two map-plane directions. A largely unexplored consequence of Nye-Kamb sliding theory is the fact that an anisotropic bed can produce a sliding velocity not parallel to the tangential traction vector. This has important consequences, since observations of non-parallel flow are often taken as indications that the shallow-ice approximation has broken down, whereas this need not be the case with an anisotropic bed.

Mathematically, this effect can be incorporated through the use of a sliding tensor. The mathematical properties of this tensor are outlined, and the correct "invariant" for the sliding law, a quadratic form, is deduced. Nye-Kamb theory for anisotropic beds is discussed. Flow on the infinite plane and the properties of surface-topography diffusion are elucidated. The properities of kinematic waves and shock waves are discussed. Kinematic waves can have a lateral component. Numerical computations of ice-sheet flow on beds with anisotropic roughness are presented, with emphasis placed on how this affects divide-ridge structure. It is suggested that cold-based ice sheets, which have an anisotropic bed affecting the shear layer, may also show non-parallelism of surface slope and velocity.
\end{abstract}

\section{INTRODUCTION}

Glacier beds are anisotropic (e.g. Hättestrand and others, 1999; personal communication from B. Hubbard, M. J. Siegert and D. McCarroll, 1998), by which is meant that the wavelengths in orthogonal directions are different. This has a number of implications, which are discussed in this paper: (i) velocity and basal-traction vectors are not parallel (Nye, 1969; there is some indirect observational evidence for this in East Antarctica, Rémy and Minster, 1997); (ii) a tensor-type relation must exist between the basal tangential traction and the sliding velocity; (iii) the "invariant" in non-linear sliding laws needs to be generalized. In addition, we discuss: (i) the predictions of Nye-Kamb theory (Nye, 1969, 1970; Kamb, 1970) for anisotropic sliding; (ii) the influence of anisotropic beds on surface response and bed-topography-surface-topography relations; and (iii) the influence of anisotropic sliding on large-scale ice-sheet flows.

\section{TENSOR SLIDING THEORY}

At their simplest, sliding laws relate two vector quantities, the basal tangential traction, $\mathbf{T}_{\mathrm{t}}$, and the basal velocity, $\mathbf{u}$, through a relationship of the form

$$
\mathbf{u}=S \mathbf{T}_{\mathrm{t}},
$$

where the smoothness $S$ is a constant or function relating to the geometry of the bed. These vectors have components $\left(T_{\mathrm{t} x}, T_{\mathrm{t} y}\right),\left(u_{x}, u_{y}\right)$, respectively. Various generalizations suggest themselves, for example

$$
\mathbf{u}=S\left(\left|\mathbf{T}_{\mathrm{t}}\right|, p_{\mathrm{e}}\right) \mathbf{T}_{\mathrm{t}},
$$

which includes a non-linear dependence on the norm of the basal traction and the scalar effective pressure $p_{\mathrm{e}}$ (e.g. Bind- schadler, 1983). A functional form for $S$ often used in icesheet modelling is

$$
S=A_{\mathrm{s}}\left|\mathbf{T}_{\mathrm{t}}\right|^{n-1},
$$

where $A_{\mathrm{s}}$ is a rate constant.

We now develop the theory for anisotropic beds. It will be seen from section 3 that a consequence of this is that the basal-velocity and basal-traction vectors are not aligned. A familiar analogy of this is the anisotropic permeability of porous media, where the potential gradient (corresponding mathematically to the tangential traction) and the Darcy velocity (corresponding to the sliding velocity) vectors are no longer aligned (Bear, 1972).

As an example of how an anisotropic bed might arise, let us consider a configuration analogous to that considered by Weertman (1957) in his study of sliding. In this analysis, Weertman's cubic obstructions are replaced by semi-ellipsoids. Suppose that the ice is flowing in a plug flow in a half 3 -space with far-field velocity $\left(u_{x}, u_{y}, 0\right)$ and consider a semi-ellipsoid with its flat edge fixed to the base of the half space, aligned with the $(x, y, z)$ frame with principal semiaxes $\left(\ell_{x}, \ell_{y}, \ell_{z}\right)$. Strain-rate magnitudes are given by

$$
\left(e_{x x}, e_{y y}\right)=\left(u_{x} / \ell_{x}, u_{y} / \ell_{y}\right),
$$

and a scale estimate of the strain-rate second invariant is $e=\left(\left(u_{x} / \ell_{x}\right)^{2}+\left(u_{y} / \ell_{y}\right)^{2}\right)^{1 / 2}$. With a Glen rheology

$$
e_{i j}=A \tau^{n-1} \tau_{i j}
$$

the longitudinal-stress magnitude is

$$
\left(\tau_{x x}, \tau_{y y}\right)=(A)^{\frac{1}{n}}\left(\left(u_{x} / \ell_{x}\right)^{2}+\left(u_{y} / \ell_{y}\right)^{2}\right)^{\frac{1}{2 n}-\frac{1}{2}}\left(u_{x} / \ell_{x}, u_{y} / \ell_{y}\right)
$$

Since we are considering perfect slip, the force, $\mathbf{F}_{\mathrm{t}}$, exerted on the bump acting in the $(x, y)$ directions is given by the 
appropriate stress times the projected area of the bump normal to the flow, i.e.

$$
\begin{aligned}
& F_{\mathrm{t} x}=\tau_{x x} \ell_{y} \ell_{z}, \\
& F_{\mathrm{t} y}=\tau_{y y} \ell_{x} \ell_{z},
\end{aligned}
$$

and using Equation (2) we find

$$
\begin{aligned}
\left(F_{\mathrm{t} x}, F_{\mathrm{t} y}\right)= & (A)^{-1 / n} \ell_{x} \ell_{y} \ell_{z}\left(\left(u_{x} / \ell_{x}\right)^{2}+\left(u_{y} / \ell_{y}\right)^{2}\right)^{(1 / n-1) / 2} \\
& \left(u_{x} / \ell_{x}^{2}, u_{y} / \ell_{y}^{2}\right)
\end{aligned}
$$

When considering scale magnitudes we can equate wavenumbers $\left(k_{x}, k_{y}\right)=\left(1 / \ell_{x}, 1 / \ell_{y}\right)$, and can therefore rewrite Equation (5) as

$$
\mathbf{T}_{\mathrm{t}}=(A)^{-1 / n} V\left(\mathbf{u}^{\mathrm{T}} \mathbf{K} \mathbf{u}\right)^{1 / 2 n-1 / 2} \mathbf{K u},
$$

where

$$
\mathbf{K}=\left[\begin{array}{cc}
k_{x}^{2} & 0 \\
0 & k_{y}^{2}
\end{array}\right]
$$

and where $V \equiv \ell_{x} \ell_{y} \ell_{z} R$, and $R$ is the number of semi-ellipsoids per unit area.

Relationship (6) exhibits some important points; (i) we expect to see a tensor-relationship between sliding velocity and basal traction; (7) is diagonal simply because we aligned the semi-axes of the ellipsoid with the coordinate frame; (ii) the invariant in the sliding law generalizes to the quadratic form $\mathbf{u}^{\mathrm{T}} \mathbf{K u}$; (iii) the components of the tensor do not depend upon the velocity field. The general truth of these statements can probably only be verified by numerical computation of ice flow over beds (e.g. Gudmundsson, 1997); however, statements (i) and (iii) are also true for Nye-Kamb sliding (see section 3), and in this case as well the tensor components depend upon the square of the wavelength/wavenumber.

We now adopt the italicized parts of statements (i), (ii) and (iii) as constitutive hypotheses and assume that they apply to anisotropic beds in general. The implications of hypothesis (i) is that the smoothness can be written as a tensor $\mathbf{S}$ such that

$$
\mathbf{u}=\mathbf{S} \mathbf{T}_{\mathrm{t}}
$$

On grounds we shall argue in more detail later, this tensor is diagonalizable, by which we mean that it has real eigenvalues. If the velocity is directed parallel to one of the corresponding eigenvectors, there will be no transverse component to the drag. The eigenvalues are termed the principal smoothnesses and the eigenvectors the principal directions of the smoothness tensor. Equation (6) is expressed in a form where the principal directions of the smoothness tensor are aligned with the coordinate axes; had the semi-ellipsoid not been aligned with the coordinate axes, the $\mathbf{K}$ matrix would have been full.

Hypothesis (ii) is of significance because, as we shall shortly see, it creates an invertible form such that we can deduce the drag from the sliding velocity. Hypothesis (iii) is the least likely to be generally true. In the rest of the section we shall suppose that

$$
\mathbf{K}=\left[\begin{array}{cc}
k_{x}^{2 m} & 0 \\
0 & k_{y}^{2 m}
\end{array}\right]
$$

where $m$ is an exponent; in the previous example and in the Nye-Kamb analysis, we find $m=1$.
The inverse form of Relationship (6) can be computed as follows. Writing (6) as

$$
\left(T_{\mathrm{t} x}, T_{\mathrm{t} y}\right)=L\left(u_{x} k_{x}^{2 m}, u_{y} k_{y}^{2 m}\right),
$$

where

$$
L \equiv(A)^{-1 / n} V\left(\mathbf{u}^{\mathrm{T}} \mathbf{K} \mathbf{u}\right)^{1 / 2 n-1 / 2},
$$

we see that we can also write (9) as

$$
\left[\begin{array}{cc}
\ell_{x}^{m} & 0 \\
0 & \ell_{y}^{m}
\end{array}\right]\left[\begin{array}{l}
T_{\mathrm{t} x} \\
T_{\mathrm{t} y}
\end{array}\right]=L\left[\begin{array}{cc}
k_{x}^{m} & 0 \\
0 & k_{y}^{m}
\end{array}\right]\left[\begin{array}{l}
u_{x} \\
u_{y}
\end{array}\right]
$$

and after multiplying the left and right sides by their respective transposes we obtain

$$
\mathbf{u}^{\mathrm{T}} \mathbf{K u}=\mathbf{T}_{\mathrm{t}}^{\mathrm{T}} \mathbf{L} \mathbf{T}_{\mathrm{t}} / L^{2}
$$

where

$$
\mathbf{L} \equiv \mathbf{K}^{-1}
$$

Substitution of this into Equation (10) gives us $L$ in terms of the traction vector,

$$
L=A^{-1} V^{n}\left(\mathbf{T}_{\mathrm{t}}^{\mathrm{T}} \mathbf{L} \mathbf{T}_{\mathrm{t}}\right)^{(1-n) / 2},
$$

and we substitute this into (9) to obtain the inverse form

$$
\left(u_{x}, u_{y}\right)=A V^{-n}\left(\mathbf{T}_{\mathrm{t}}^{\mathrm{T}} \mathbf{L} \mathbf{T}_{\mathrm{t}}\right)^{(n-1) / 2}\left(T_{\mathrm{t} x} \ell_{x}^{2 m}, T_{\mathrm{t} y} \ell_{y}^{2 m}\right) .
$$

The scale-model generalization of Equation (1) is thus

$$
\mathbf{S}=A V^{-n}\left(\mathbf{T}_{\mathrm{t}}^{\mathrm{T}} \mathbf{L} \mathbf{T}_{\mathrm{t}}\right)^{(n-1) / 2} \mathbf{L},
$$

where $\mathbf{S}$ is the tensor equivalent of $S$. We shall see in the next section that Nye-Kamb theory gives this functional form for the special case $n=1$ and with small bed undulations.

The above argument has supposed that the $\mathbf{L}$ and $\mathbf{K}$ tensors are diagonal. However, in the above example the diagonal form arises from the fact that we have aligned the axes of the ellipsoid with the coordinate axes. A more general form, where the "grain" of the landscape is not aligned with the coordinate axes, will result in a full smoothness tensor. If we define the principal smoothnesses $S_{1}$ and $S_{2}$ to be at angle $\theta$ to the coordinate axes, we find the smoothness tensor to be

$$
\mathbf{S}=\left[\begin{array}{cc}
S_{1} \cos ^{2} \theta+S_{2} \sin ^{2} \theta & \cos \theta \sin \theta\left(S_{1}-S_{2}\right) \\
\cos \theta \sin \theta\left(S_{1}-S_{2}\right) & S_{1} \sin ^{2} \theta+S_{2} \cos ^{2} \theta
\end{array}\right]
$$

after applying the usual tensor-transformation rules. For isotropic sliding we can, without loss of generality, take $(\mathbf{K}, \mathbf{L})=(\mathbf{I}, \mathbf{I})$, and we then obtain the usual sliding invariant used in glaciology.

The quadratic-form construction depends on the existence of the smoothness tensor, and is a form which permits inversion of the sliding relationship, since the forms $\mathbf{T}_{\mathrm{t}}^{\mathrm{T}} \mathbf{L} \mathbf{T}_{\mathrm{t}}$ and $\mathbf{u}^{\mathrm{T}} \mathbf{K u}$ maintain a constant ratio irrespective of the orientation of the coordinate axes. The dissipation is given by $\mathbf{T}_{\mathrm{t}} \cdot \mathbf{u}=\mathbf{T}_{\mathrm{t}} \cdot \mathbf{S} \mathbf{T}_{\mathrm{t}}$, which implies that the tensor $\mathbf{S}$ should be positive definite; if it were not energy production could be negative.

Finally, we show that the tensor $\mathbf{S}$ is symmetric. A positivedefinite matrix is one where the eigenvalues (principal smoothnesses) are positive. This means that we can write $\mathbf{S}=$ $\mathbf{E} \Lambda \mathbf{E}^{-1}$, where $\Lambda$ is the diagonal matrix of eigenvalues and $\mathbf{E}$ is the matrix of eigenvectors. Since the matrix $\Lambda$ is also a smoothness matrix in a rotated frame, by using the usual transformation rules we can write $\mathbf{S}=\mathbf{R} \Lambda \mathbf{R}^{\mathrm{T}}$, where $\mathbf{R}$ is the (orthonormal) rotation matrix. This means that the eigenvectors are an orthonormal matrix and we can write $\mathbf{S}=$ $\mathbf{E} \Lambda \mathbf{E}^{\mathrm{T}}$. This can be written as $\mathbf{S}=\mathbf{F F}^{\mathrm{T}}$ where $\mathbf{F}=\mathbf{E} \Lambda^{1 / 2}$. 
Since the product of a real matrix and its transpose is symmetric, this shows that the smoothness tensor is symmetric.

\section{NYE-KAMB THEORY}

Nye-Kamb theory (Nye, 1969, 1970; Kamb, 1970) considers the case where a block of ice is moved, with perfect slip, over a flat surface, at a prescribed velocity $\left(u_{\mathrm{b}}, 0,0\right)$. Perturbative undulations in the bed are introduced. These create a firstorder velocity field which vanishes at large distance from the surface; the ice velocity therefore remains $\left(u_{\mathrm{b}}, 0,0\right)$ at large distance. These undulations introduce a drag, which is a second-order quantity as it is a multiple of two firstorder quantities, the normal stress and the bed slope. Where slip is perfect, there are no contributions from the secondorder stress field to the second-order drag, and we therefore do not need to compute the second-order stress field to compute the second-order drag. We now treat Nye-Kamb sliding over an anisotropic bed. Three-dimensional flow has been treated by Nye $(1969,1970)$ who in particular computed the "transverse drag" for the case where the bedprofile autocorrelation function is anisotropic. We consider a simpler case (where two washboards are superimposed), emphasizing the tensorial nature of the solution and the dependence of the tensor components on the components of the wavelength vector; neither of these features are very obvious in Nye's work. We ignore regelation.

In the rest of the paper we consider perturbed and unperturbed velocity fields. When we are considering a threedimensional-velocity field, we denote it $\mathbf{v}$ with components $\left(v_{x}, v_{y}, v_{x}\right)$. Sometimes we wish to restrict consideration to a horizontal-velocity field $\mathbf{u}$ which has components $\left(u_{x}, u_{y}\right)$ $\equiv\left(v_{x}, v_{y}\right)$. When we consider this horizontal-velocity-field vector and wish to consider the vertical velocity as well, we denote it $w$; by definition $w \equiv v_{z}$.

Now we consider a perturbed half-space $\tilde{z}>D=$ $\hat{D} \exp (-i \mathbf{k} \cdot \mathbf{r}), \mathbf{k}=\left(k_{x}, k_{y}\right), \mathbf{r}=(x, y)$ and specifically consider the case $\hat{D}=\mu \hat{D}_{1}$, where $\mu$ is a small parameter. In this half-space ice is moving with perfect slip over the base. Following Nye (1970) we define a new coordinate $z=$ $\tilde{z}-\hat{D} \exp (-i \mathbf{k} \cdot \mathbf{r})$, and obtain the field equations

$$
\eta \nabla^{2} \mathbf{v}_{1}=\nabla p_{1}
$$

where $\mathbf{v}_{1}$ is the perturbed three-dimensional vector field, $p_{1}$ is the perturbed pressure and $\eta$ is the viscosity of ice. The zeroth-order solution, corresponding to $\mu=0$, is

$$
\begin{aligned}
& \mathbf{v}_{0}=\left(u_{b}, 0,0\right), \\
& p_{0}=0 .
\end{aligned}
$$

Boundary conditions are

$$
\begin{aligned}
\mathbf{v}_{1} & \rightarrow 0, \quad p \rightarrow 0 \quad \text { as } \quad z \rightarrow \infty \\
\partial_{z} \mathbf{u}_{1}+\nabla_{H} w_{1} & =0 \text { (perfect slip) } \\
w_{1} & =\mathbf{u}_{0} \cdot \nabla D_{1}(\text { basal kinematical condition) }
\end{aligned}
$$

where $\nabla_{\mathrm{H}}$ is the horizontal-divergence operator. (For functions of $x$ and $y$ only, we do not in general distinguish between $\nabla_{\mathrm{H}}$ and $\nabla$ ). These equations possess the solution (cf. Nye, 1969, 1970)

$$
\begin{aligned}
v_{1 x} & =\frac{i k_{x}}{|\mathbf{k}|} \frac{P z}{2 \eta} \exp (-|\mathbf{k}| z-i \mathbf{k} \cdot \mathbf{r}), \\
v_{1 y} & =\frac{i k_{y}}{|\mathbf{k}|} \frac{P z}{2 \eta} \exp (-|\mathbf{k}| z-i \mathbf{k} \cdot \mathbf{r}), \\
v_{1 z} & =\frac{P}{2 \eta}\left(\frac{1}{|\mathbf{k}|}+z\right) \exp (-|\mathbf{k}| z-i \mathbf{k} \cdot \mathbf{r}), \\
p_{1} & =P \exp (-|\mathbf{k}| z-i \mathbf{k} \cdot \mathbf{r})
\end{aligned}
$$

where

$$
P \equiv-2 i \eta|\mathbf{k}| \mathbf{k} \cdot \mathbf{u}_{0} \hat{D}_{1}
$$

Use of the basal kinematical condition shows that

$$
T_{1}^{n}=-2 i \eta \mathbf{k} \cdot \mathbf{u}_{0}|\mathbf{k}| \hat{D}_{1} \exp (-i \mathbf{k} \cdot \mathbf{r}),
$$

where we have used

$$
\nabla D_{1}=-i \mathbf{k} \hat{D}_{1} \exp (-i \mathbf{k} \cdot \mathbf{r})
$$

and to obtain the average drag per unit area we use the second-order approximation

$$
\begin{aligned}
\mathbf{T}_{2}= & \frac{k_{x} k_{y}}{4 \pi^{2}} \int_{0}^{2 \pi / k_{x}} \int_{0}^{2 \pi / k_{y}} T_{1}^{n} \nabla D_{1} \mathrm{~d} y \mathrm{~d} x \\
= & \frac{k_{x} k_{y}}{2 \pi^{2}} \eta \mathbf{k}\left(\mathbf{k} \cdot \mathbf{u}_{0}\right)|\mathbf{k}| \hat{D}_{1}^{2} \times \\
& \int_{0}^{2 \pi / k_{x}} \int_{0}^{2 \pi / k_{y}} \exp (-2 i \mathbf{k} \cdot \mathbf{r}) \mathrm{d} y \mathrm{~d} x, \\
= & \eta \mathbf{k}\left(\mathbf{k} \cdot \mathbf{u}_{0}\right)|\mathbf{k}| \hat{D}_{1}^{2} .
\end{aligned}
$$

We can express this in tensor form through

$$
\begin{aligned}
\mathbf{T}_{2} & =\frac{1}{2} \eta|\mathbf{k}| \hat{D}_{1}^{2} K \mathbf{u}_{0}, \\
\mathbf{K} & =\left[\begin{array}{ll}
k_{x}^{2} & k_{x} k_{y} \\
k_{x} k_{y} & k_{y}^{2}
\end{array}\right] .
\end{aligned}
$$

The matrix $\mathbf{K}$ has eigenvalues $(\mathbf{k} \cdot \mathbf{k}, 0)$ with corresponding orthogonal principal directions $\left(\left[\begin{array}{ll}k_{x} / k_{y} & 1\end{array}\right],\left[\begin{array}{ll}-k_{y} / k_{x} & 1\end{array}\right]\right)$. The orthogonality arises from the symmetry of the matrix. The singularity of the matrix, as evinced by the zero eigenvalue, indicates that we can deduce the drag from the sliding velocity, but that we cannot deduce the sliding velocity from the drag. It stems from the fact that the function $\exp (-i \mathbf{k} \cdot \mathbf{r})$ is an angled washboard and that the drag parallel to the axis of the washboard must be zero for perfect slip.

In this linear case, more complicated topographies can be constructed by superposing $N$ different washboard topographies. Let us number indices $(j, \ell) \in(1, N)$. Use of the basal kinematic condition shows that the normal traction is given by

$$
T_{j 1}^{n}=-2 i \eta \mathbf{k}_{j} \cdot \mathbf{u}_{0}\left|\mathbf{k}_{j}\right| \hat{D}_{j 1} \exp \left(-i \mathbf{k}_{j} \cdot \mathbf{r}\right),
$$

and the average drag per unit area is given by

$$
\begin{aligned}
& \mathbf{T}_{2}=\frac{1}{X Y} \sum_{j} \sum_{\ell} \int_{0}^{X} \int_{0}^{Y} T_{j 1}^{n} \nabla D_{\ell 1} \mathrm{~d} y \mathrm{~d} x \\
&=\frac{2 \eta}{X Y} \sum_{j} \sum_{\ell} \int_{0}^{X} \int_{0}^{Y} \hat{D}_{j 1} \mathbf{k}_{j} \cdot \mathbf{u}_{0}\left|\mathbf{k}_{j}\right| \exp \left(-i \mathbf{k}_{j} \cdot \mathbf{r}\right) \times \\
& \hat{D}_{\ell 1} \mathbf{k}_{\ell} \exp \left(-i \mathbf{k}_{\ell} \cdot \mathbf{r}\right) \mathrm{d} y \mathrm{~d} x, \\
& X=\operatorname{lcm}\left(\left|2 \pi / k_{j x}\right|,\left|2 \pi / k_{\ell x}\right|\right), \\
& Y=\operatorname{lcm}\left(\left|2 \pi / k_{j y}\right|,\left|2 \pi / k_{\ell y}\right|\right),
\end{aligned}
$$

where $\mathrm{lcm}$ indicates the least common multiple. 

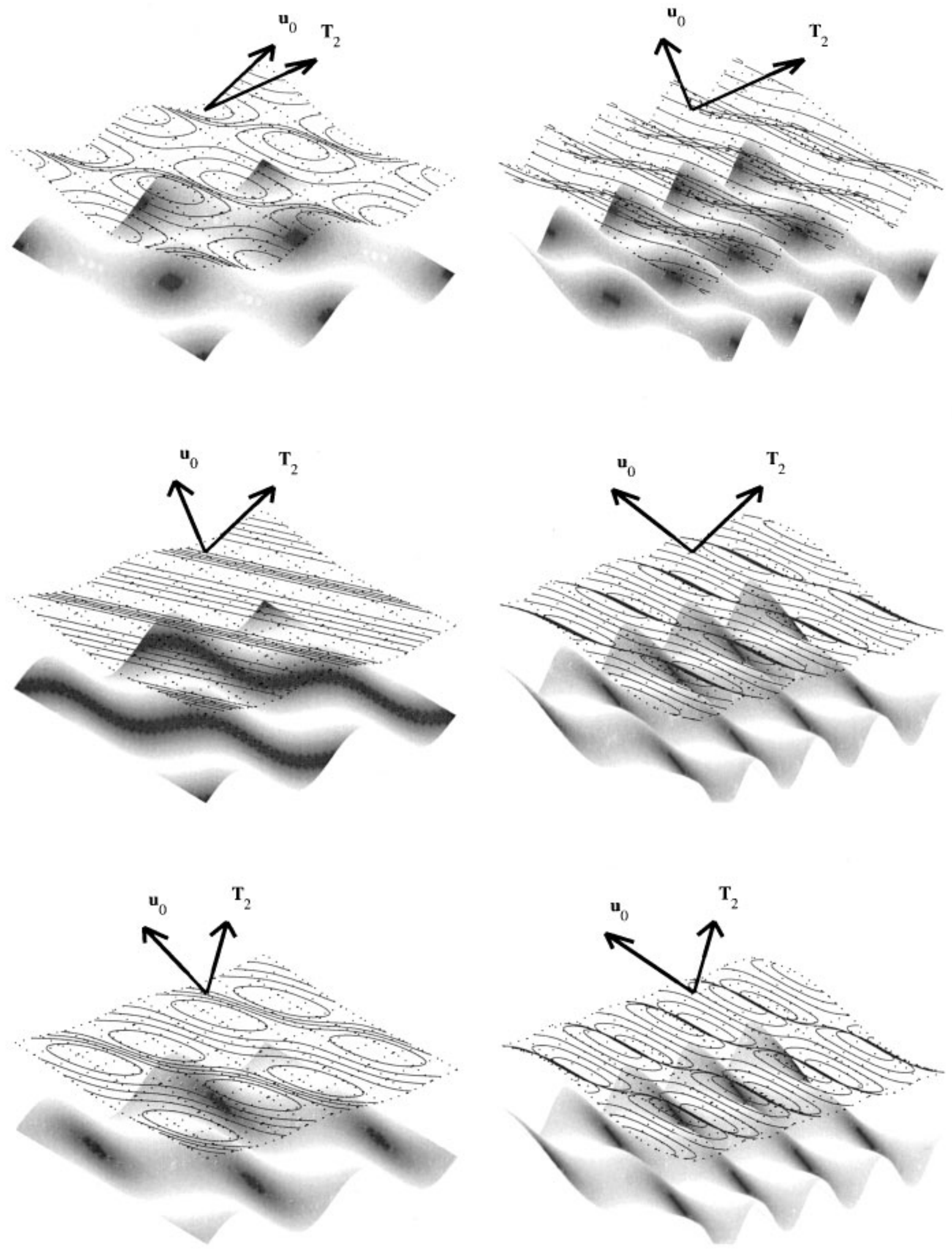

Fig. 1. Anisotropic flow with perfect slip around bumps of wavelength much shorter than the ice thickness (Nye-Kamb configuration). The relationship between basal topography, interfacial-pressure distribution, zeroth-order velocity $\mathbf{u}_{0}$ and the drag vector $\mathbf{T}_{2}$. The two columns correspond to different basal topographies, while the rows correspond to different orientations of the drag vector. These different orientations cause the changing orientation of the velocity vector. If the drag vector were parallel to either of the principal axes of the smoothness tensor, corresponding to the axes of the diagram, the velocity vector would be parallel to the traction vector. Basal topography is indicated by the shape of the lower, grey surface. The pressure is indicated three ways; by the shading on the basal topography, with darker indicating higher presure; by the shape of the upper mesh, and by the three-dimensional contouring on this mesh. 
A topography containing hummocks and hollows can be created by superposing two washboard topographies defined by wavenumbers $\left(k_{x}, k_{y}\right)$ and $\left(k_{x},-k_{y}\right), \hat{D}_{j 1}=\hat{D}_{\ell 1}=\frac{1}{2} \hat{D}_{1}$, which means that the amplitude of the basal topography is $\hat{D}_{1}$. In this case, we find

$$
\begin{aligned}
\mathbf{T}_{2} & =\frac{k_{x} k_{y}}{4 \pi^{2}} \sum_{j} \sum_{\ell} \int_{0}^{2 \pi / k_{x}} \int_{0}^{2 \pi / k_{y}} T_{j 1}^{n} \nabla D_{\ell 1} \mathrm{~d} y \mathrm{~d} x \\
& =\eta \frac{\hat{D}_{1}^{2}}{2}|\mathbf{k}|\left[\begin{array}{cc}
k_{x}^{2} & 0 \\
0 & k_{y}^{2}
\end{array}\right] \mathbf{u}_{0} .
\end{aligned}
$$

This tensor is not singular for non-zero wavenumbers, and can be readily inverted to create the inverse form

$$
\mathbf{u}_{0}=\frac{2}{\eta|\mathbf{k}| \hat{D}_{1}^{2}}\left[\begin{array}{cc}
1 / k_{x}^{2} & 0 \\
0 & 1 / k_{y}^{2}
\end{array}\right] \mathbf{T}_{2} .
$$

Note that the average drag and the zeroth-order velocity are not parallel even in this simple example; this is a result of the additional stresses in the ice caused by the presence of the bed topography. The basic solution of glacier sliding thus gives anisotropic sliding. In this construction we have aligned the principal axes of the sliding tensor with the coordinate axes, but it could equally be aligned with the velocity or the drag. In such a case, the sliding tensor would in general contain non-zero off-diagonal elements. The diagonal form arises from the fact that the washboard axes subtend the same absolute angle with the axes.

Some examples are plotted in Figure 1. The two columns correspond to different basal topography, while the rows correspond to the average drag vector, which is pointed increasingly obliquely to the $x$ axis. In the left column, the wavelength ratios are $2: 1$, while in the right column, they are $4: 1$. This will cause the ratio of the principal roughnesses (inverse smoothnesses) to be $4: 1$ and $16: 1$ respectively. It can be seen that flow is directed along the longer wavelength direction for relatively small deflections of the drag vector from the $x$ axis, and that this effect is particularly marked for the more anisotropic case. Pressure maxima reside on the upstream side of the bumps, their orientation seeming to be more related to the velocity direction rather than the drag direction.

\section{SURFACE EFFEGTS}

Here we extend to the case of anisotropic sliding analyses due to Nye (1959) and to Budd (1970) which show: (i) how disturbances in the surface profile decay to the zeroth order profile; and (ii) how variations in the bed profile affect the surface profile. In both cases, we consider flow down an infinitely long and broad plane, with gravity components given by $g(\varepsilon, 0,-1)$. We are assuming $\varepsilon$ to be small, but it is not an aspect ratio and not a perturbation parameter. Wavelengths of undulations which give rise to surface effects are much larger than the ice thickness in this analysis and the mechanics can thus be described by the shallow-ice approximation (Hutter, 1983). However anisotropic sliding arises from bedrock undulations with wavelength much smaller than the ice thickness so that the effect of these undulations is not noticeable at the surface. We are thus dealing with two distinct limits, one of which gives rise to the surface effects and the other of which gives rise to the anisotropic sliding.

We expand $H=H_{0}+\nu H_{1}$ etc., where $\nu$ is a small parameter. At zeroth order, the basal shear stress is given by

$$
T_{0 x}=\rho_{\mathrm{i}} g H \varepsilon, \quad T_{0 y}=0,
$$

where $\rho_{\mathrm{i}}$ is the density of ice, $g$ is the acceleration due to gravity and $H$ is the thickness of the ice. There is subtlety here; $\mathbf{T}_{0}$ here refers to the expansion in the small parameter $\nu$. This resistance arises from short-wavelength perturbations, and corresponds to the second-order drag $\mathbf{T}_{2}$ obtained from perturbing in parameter $\mu$ discussed in section 3 .

The sliding velocity is given by

$$
\mathbf{u}_{0}=A_{\mathrm{s}}\left(\mathbf{T}_{0}^{\mathrm{T}} \mathbf{L} \mathbf{T}_{0}\right)^{(n-1) / 2} \mathbf{L} \mathbf{T}_{0},
$$

(see Equation (8)) where $\mathbf{L}$ is the tensor defined in Equation (11). This shows that the zeroth-order velocity need not be in the $x$ direction if the principal smoothnesses are not aligned with the coordinate axes.

The first-order basal tractions are

$$
\begin{aligned}
& T_{1 x}=T_{0 x}\left(H_{1} / H_{0}-\partial_{x} H_{1} / \varepsilon\right), \\
& T_{1 y}=-T_{0 x} \partial_{y} H_{1} / \varepsilon,
\end{aligned}
$$

and the first-order velocity vector becomes

$$
\begin{aligned}
& \mathbf{u}_{1}=A_{s}\left|\mathbf{T}_{0}\right|^{n-1} \mathbf{L} T_{0} x \times \\
& {\left[\begin{array}{c}
L_{x} x(n-1)\left(H_{1} / H_{0}-\partial_{x} H_{1} / \varepsilon\right)+\left(H_{1} / H_{0}-\partial_{x} H_{1} / \varepsilon\right) \\
-\partial_{y} H_{1} / \varepsilon-L_{x} y(n-1) \partial_{y} H_{1} / \varepsilon
\end{array}\right]}
\end{aligned}
$$

At first order the continuity equation is

$$
\partial_{\mathrm{t}} H_{1}=-H_{0} \nabla \cdot \mathbf{u}_{1}-\nabla H_{1} \cdot \mathbf{u}_{0} .
$$

To proceed, we define a surface wavenumber $\mathbf{k}^{\mathrm{s}}$ and write

$$
\begin{aligned}
H_{1} & =\hat{H}_{1} \exp \left(-i \mathbf{k}^{\mathrm{s}} \cdot \mathbf{r}\right), \quad \nabla H_{1}=-i \mathbf{k}^{\mathrm{s}} \hat{H}_{1} \exp \left(-i \mathbf{k}^{\mathrm{s}} \cdot \mathbf{r}\right), \\
\mathbf{u}_{1} & =U_{0} \mathbf{L}\left[\begin{array}{c}
\nu_{x}\left(\hat{H}_{1} / H_{0}+i k_{x}^{\mathrm{s}} \hat{H}_{1} / \varepsilon\right) \\
i \nu_{y} k_{y}^{\mathrm{s}} \hat{H}_{1} / \varepsilon
\end{array}\right] \exp \left(-i \mathbf{k}^{\mathrm{s}} \cdot \mathbf{r}\right), \\
\nabla \cdot \mathbf{u}_{1} & =-i U_{0}\left(\mathbf{k}^{\mathrm{s}}\right)^{\mathrm{T}} \mathbf{L}\left[\begin{array}{c}
\nu_{x}\left(\hat{H}_{1} / H_{0}+i k_{x}^{\mathrm{s}} \hat{H}_{1} / \varepsilon\right) \\
i \nu_{y} k_{y}^{\mathrm{s}} \hat{H}_{1} / \varepsilon
\end{array}\right] \exp \left(-i \mathbf{k}^{\mathrm{s}} \cdot \mathbf{r}\right), \\
\frac{\mathrm{d} \hat{H}_{1}}{\mathrm{~d} t} & =Q_{0}\left(\mathbf{k}^{\mathrm{s}}\right)^{\mathrm{T}} \mathbf{L}\left[\begin{array}{c}
i\left(\nu_{x}+1\right) / H_{0}-\nu_{x} k_{x}^{\mathrm{s}} / \varepsilon \\
-\nu_{y} k_{y}^{\mathrm{s}} / \varepsilon
\end{array}\right] \hat{H}_{1},
\end{aligned}
$$

where we define the scalars

$$
\begin{aligned}
U_{0} & \equiv A_{\mathrm{s}}\left|\mathbf{T}_{0}^{\mathrm{T}} \mathbf{L} \mathbf{T}_{0}\right|^{n-1} T_{0 x} \\
Q_{0} & \equiv H_{0} U_{0} \\
\nu_{x} & \equiv L_{x x}(n-1)+1 \\
\nu_{y} & \equiv L_{x y}(n-1)+1 .
\end{aligned}
$$

\subsection{Decay of surface disturbances}

The decay-rate constant is given by the real part of the ratio $\left(\mathrm{d} \hat{H}_{1} / \mathrm{d} t\right) / \hat{H}_{1}$, which is

$$
-Q_{0}\left(\mathbf{k}^{\mathrm{s}}\right)^{\mathrm{T}} \mathbf{L}\left[\nu_{x} k_{x}^{\mathrm{s}}, \nu_{y} k_{y}^{\mathrm{s}}\right]^{\mathrm{T}} / \varepsilon
$$

We can prove stability as follows. Let us rotate the axes into a coordinate system $\left(x^{\prime}, y^{\prime}\right)$ determined by the principal directions of $\mathbf{L}$. This implies that the transformed tensor $\mathbf{L}^{\prime}$ is diagonal, and we can see immediately that the decay rate is

$$
-Q_{0}\left(k_{x}^{\mathrm{s}}\right)^{2} L_{x x}^{\prime}\left(L_{x x}^{\prime}(n-1)+1\right) / \varepsilon<0
$$

since the principal smoothness $L_{x x}^{\prime}>0$ by the positive-definiteness property. The imaginary component can be written $\left(\nu_{x}+1\right) U_{0}\left(k_{x}^{\mathrm{s}} L_{x x}+L_{x y} k_{y}^{\mathrm{s}}\right)$. For one-dimensional flow over an isotropic bed, $k_{y} \rightarrow 0$ and without loss of generality we can write $\mathbf{L}=\mathbf{I}$, which allows us to retrieve the wellknown one-dimensional results.

Wave velocity (i.e. of a washboard surface wave) in the $x$ 


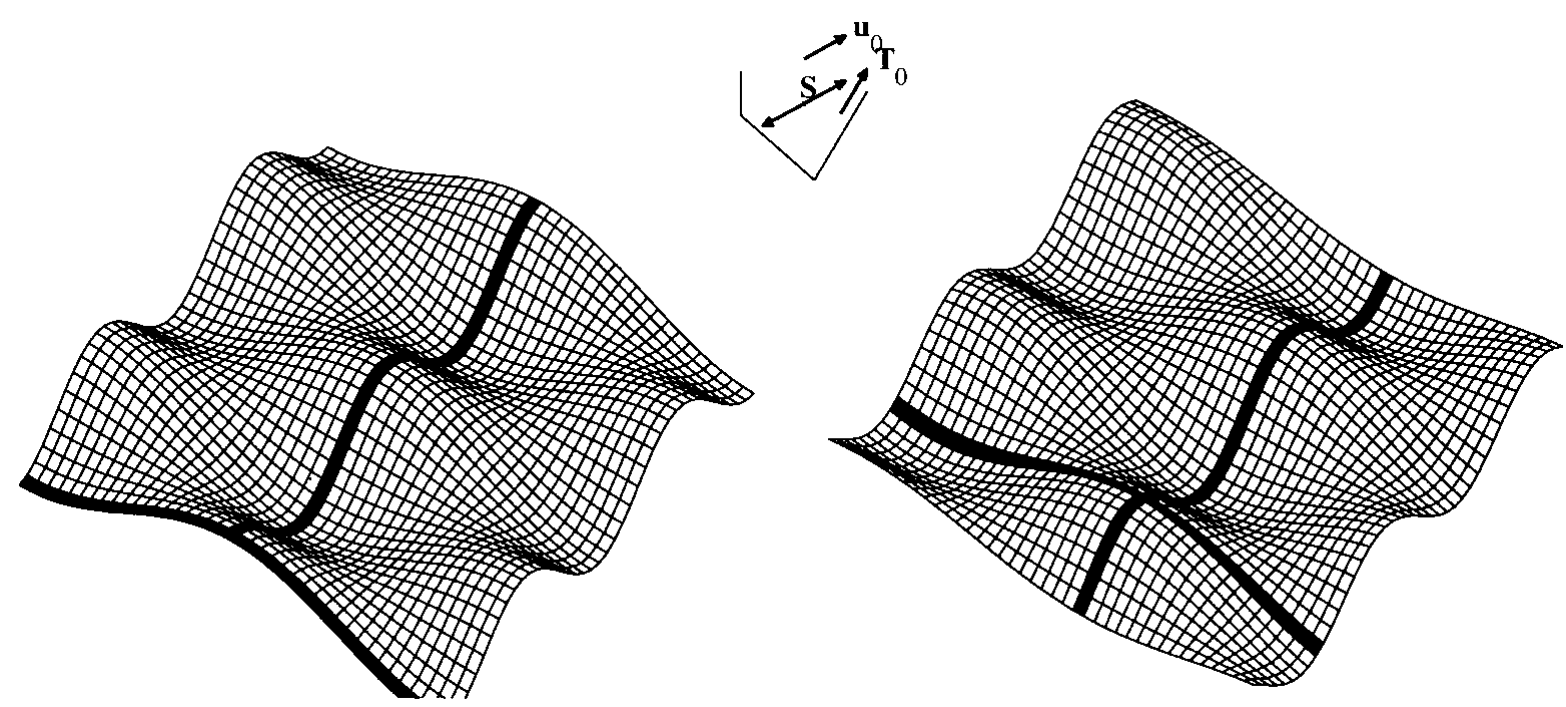

Fig. 2. Illustrating the motion of kinematic waves of wavelength greater than the ice thickness. The surface represents the glacier surface and the dark lines are included to demonstrate the wave motion. Note how anisotropic flow leads to lateral wave motion; the lateral velocity depends on the wavelength, i.e. it is dispersive, unlike the downstream velocity.

direction is given by $\left(\nu_{x}+1\right) U_{0}\left(L_{x x}+L_{x y} k_{y}^{\mathrm{s}} / k_{x}^{\mathrm{s}}\right)$, while the $x$ velocity of the plane (washboard) wave of opposite orientation to the $y$ axis is given by $\left(\nu_{x}+1\right) U_{0}$ $\left(L_{x x}-L_{x y} k_{y}^{\mathrm{s}} / k_{x}^{\mathrm{s}}\right)$. Both waves will remain plane waves oriented at the same angle, moving at different velocities. Since their superposition is hummocks and hollows oriented with the $x$ and $y$ axes, we can anticipate that these features will migrate laterally if $L_{x y} \neq 0$, which of course happens when the principal smoothnesses are not aligned with the coordinate axes/downstream direction. This is illustrated in Figure 2. In particular, we are interested in the ratio of the lateral- and longitudinal-wave-migration velocities, how this relates to the lateral and longitudinal components of the zeroth-order ice-flow velocity and how this wavevelocity ratio is affected by the wavenumbers.

\subsection{Bedrock-surface relationships}

We now wish to investigate the steady surface response to bedrock perturbations (Hutter, 1983, chapter 4). This is carried out for wavelengths greater than the ice thickness, while the wavelengths which determine the sliding resistance are much less than the ice thickness. We suppose that the bed profile is given by $z=b_{1}$. The first-order stresses are given by

$$
\begin{aligned}
& T_{1 x}=T_{0 x}\left(H_{1} / H_{0}-\partial_{x}\left(H_{1}+b_{1}\right) / \varepsilon\right), \\
& T_{1 y}=-T_{0 x} \partial_{y}\left(H_{1}+b_{1}\right) / \varepsilon
\end{aligned}
$$

and the first-order velocity vector becomes

$$
\mathbf{u}_{1}=A_{\mathrm{s}}\left|\mathbf{T}_{0}\right|^{n-1} \mathbf{L} T_{0 x}\left[\begin{array}{c}
\nu_{x}\left(H_{1} / H_{0}-\partial_{x}\left(H_{1}+b_{1}\right) / \varepsilon\right) \\
-\nu_{y} \partial_{y}\left(H_{1}+b_{1}\right) / \varepsilon
\end{array}\right]
$$

To proceed, we assume the bedrock topography is defined by a wavenumber vector $\mathbf{k}^{\mathrm{b}}$ and assume that under the linearity assumption this will be the wavelength of the surface topography. Thus,

$$
\begin{aligned}
b_{1} & =\hat{b}_{1} \exp \left(-i \mathbf{k}^{\mathrm{b}} \cdot \mathbf{r}\right), \nabla b_{1}=-i \mathbf{k}^{\mathrm{b}} \hat{b}_{1} \exp \left(-i \mathbf{k}^{\mathrm{b}} \cdot \mathbf{r}\right), \\
H_{1} & =\hat{H}_{1} \exp \left(-i \mathbf{k}^{\mathrm{b}} \cdot \mathbf{r}\right), \nabla H_{1}=-i \mathbf{k}^{\mathrm{b}} \hat{H}_{1} \exp \left(-i \mathbf{k}^{\mathrm{b}} \cdot \mathbf{r}\right),
\end{aligned}
$$

and we can readily deduce that

$$
\begin{aligned}
\mathbf{u}_{1}= & U_{0} \mathbf{L} \times \\
& {\left[\begin{array}{c}
\nu_{x}\left(\hat{H}_{1} / H_{0}+i k_{x}^{\mathrm{b}}\left(\hat{H}_{1}+\hat{b}_{1}\right) / \varepsilon\right) \\
i \nu_{y} k_{y}^{\mathrm{s}}\left(\hat{H}_{1}+\hat{b}_{1}\right) / \varepsilon
\end{array}\right] \exp \left(-i \mathbf{k}^{\mathrm{b}} \cdot \mathbf{r}\right), } \\
\nabla \cdot \mathbf{u}_{1}= & -i U_{0}\left(\mathbf{k}^{\mathrm{b}}\right)^{\mathrm{T}} \mathbf{L} \times \\
& {\left[\begin{array}{c}
\nu_{x}\left(\hat{H}_{1} / H_{0}+i k_{x}^{\mathrm{b}}\left(\hat{H}_{1}+\hat{b}_{1}\right) / \varepsilon\right) \\
i \nu_{y} k_{y}^{\mathrm{b}}\left(\hat{H}_{1}+\hat{b}_{1}\right) / \varepsilon
\end{array}\right] \exp \left(-i \mathbf{k}^{\mathrm{b}} \cdot \mathbf{r}\right), }
\end{aligned}
$$

and the steady condition is

$$
\begin{gathered}
\left(\mathbf{k}^{\mathrm{b}}\right)^{\mathrm{T}} \mathbf{L}\left[\begin{array}{c}
\left(\nu_{x}+1\right)\left(1 / H_{0}+i k_{x}^{\mathrm{b}} / \varepsilon\right) \\
i \nu_{y} k_{y}^{\mathrm{b}} / \varepsilon
\end{array}\right] \hat{H}_{1} \\
=-\left(\mathbf{k}^{\mathrm{b}}\right)^{\mathrm{T}} \mathbf{L}\left[\begin{array}{c}
i \nu_{x} k_{x}^{\mathrm{b}} / \varepsilon \\
i \nu_{y} k_{y}^{\mathrm{b}} / \varepsilon
\end{array}\right] \hat{b}_{1} .
\end{gathered}
$$

This equation applies to the case of the angled washboard, i.e. where the bed is a washboard with axes angled to the downstream, $x$ direction. The magnitude of the surface effect is determined by $\operatorname{Re}\left(\hat{H}_{1}\right)$, while $\operatorname{Im}\left(\hat{H}_{1}\right)$ determines the phasing relative to the bedrock topography.

The superposition of two angled washboards leads to a hummocks and hollows topography, and solution for the elevation may be found by superposing solutions for $\hat{H}_{1}$. Some examples are plotted in Figure 3. The traction vector is aligned with the $x$ axis. The two columns correspond to different basal topography, while the rows correspond to different alignments of the principal smoothnesses, the major one being pointed increasingly obliquely to the $x$ axis. The resulting zeroth-order velocity is indicated. Where the long-wavelength topography (shown on the figure) is anisotropic and aligned orthogonal to the traction direction, surface maxima reside upslope of the basal maxima, but when the long-wavelength basal topography is isotropic, surface maxima reside upflow of the basal maxima. If the sliding were isotropic, upflow and upslope would be in the same direction under the shallow-ice approximation, but anisotropic sliding permits them to be different when the slope 

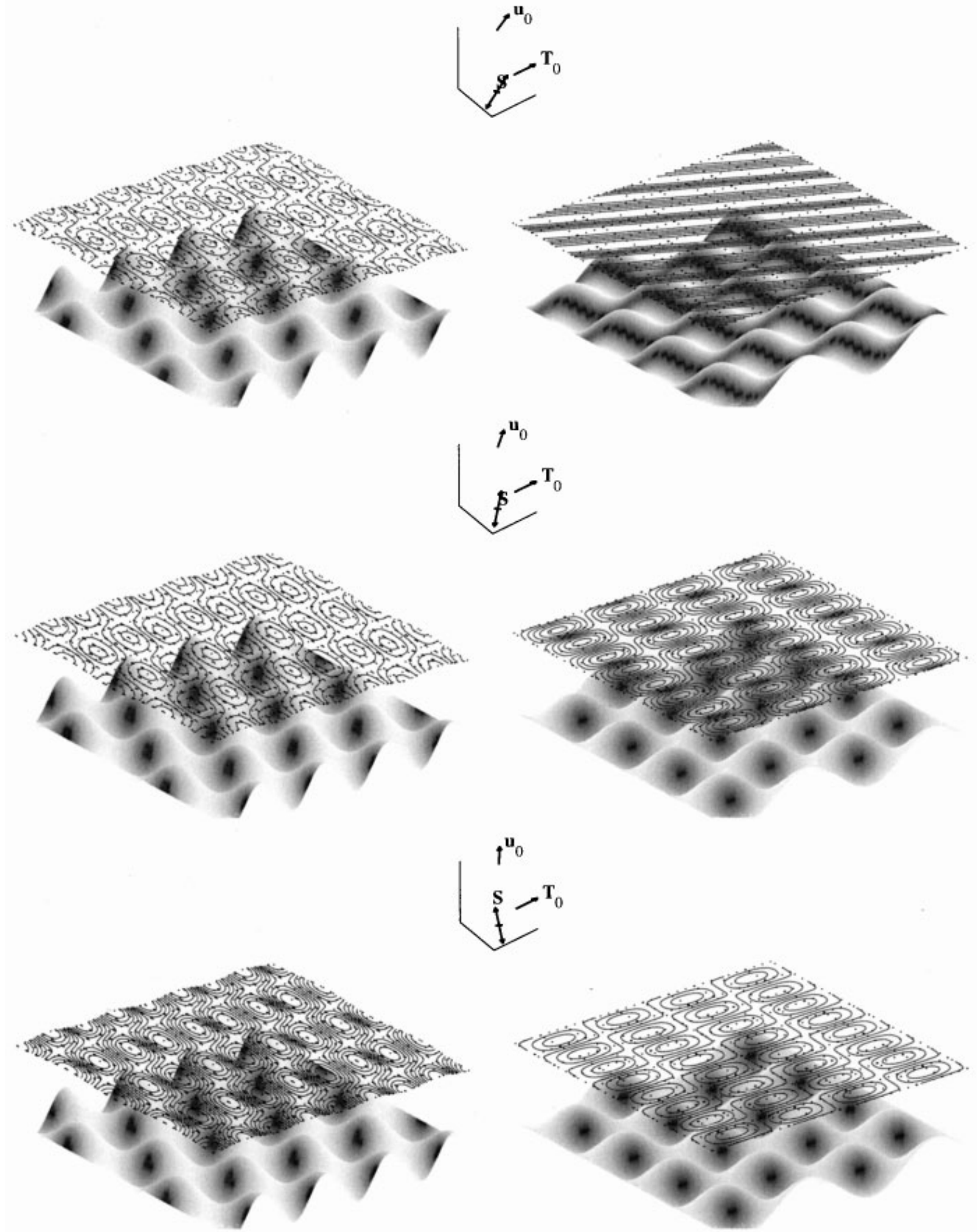

Fig. 3. The relationship between basal topography, and ice-surface elevation for wavelengths greater than the ice thickness (the shallow-ice approximation). The two columns correspond to different basal topographies, while the rows correspond to different orientations of the principal smoothnesses. Basal topgraphy is indicated by the shape of the lower, grey surface. The surface is indicated three ways; by the shading on the basal topography, with darker indicating greater ice-surface elevation; by the shape of the upper mesh; and by the three-dimensional contouring on this mesh. The small axes indicate the direction of the traction (parallel to the $x$ axis), the zeroth-order sliding velocity and the principal smoothnesses. The ratio of principal smoothnesses was 10:1. 
and velocity are not aligned with one of the principal axes of the smoothness tensor.

\subsection{Shock velocities}

In valley glaciers, the basic form of the evolution equation for ice thickness is hyperbolic and kinematic waves will form shocks, where there is a jump in the thickness (Fowler, 1982). These shocks will be severely smoothed by diffusion (Jóhannesson, 1992), but the possibility of smoothed shock-like form existing remains. The shock is also of mathematical interest as it represents a limiting non-linear behaviour.

The shock speed $v$ is given by the Rankine-Hugoniot condition

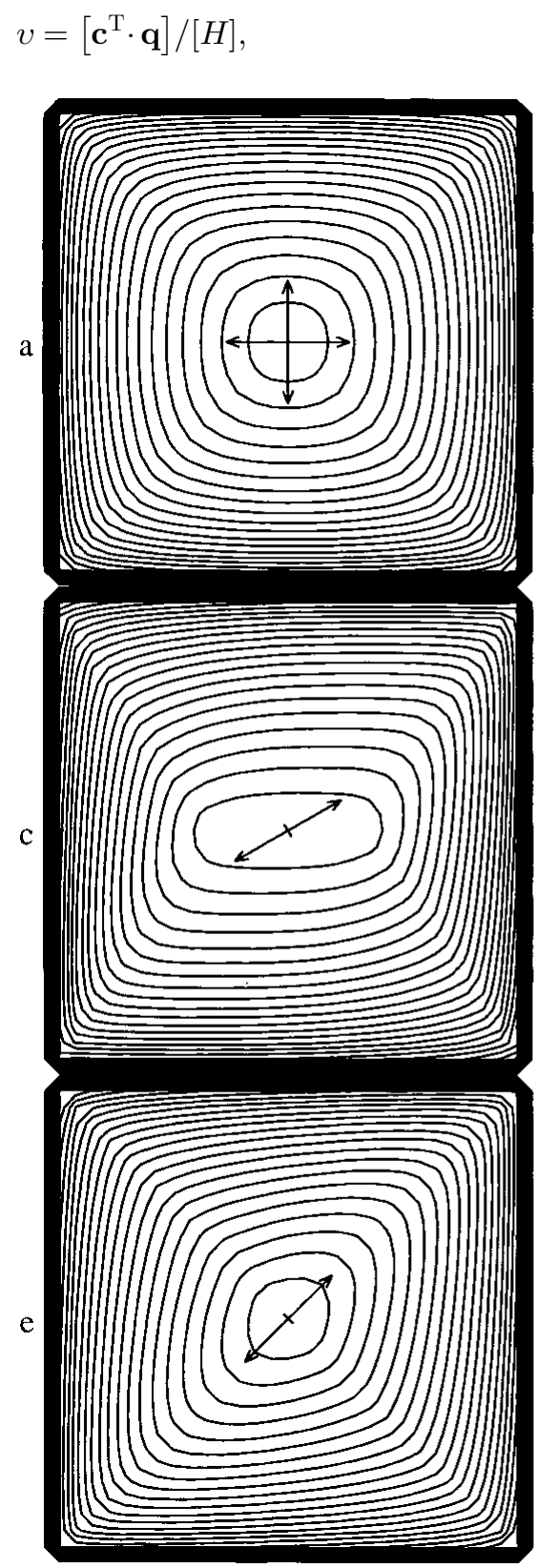

where $\mathbf{c}$ is the unit vector normal to the shock. If the traction vector is aligned with the $x$ axis, the flux is given by

$$
\mathbf{q}=A_{\mathrm{s}}\left(T_{x}^{2} L_{x x}\right)^{(n-1) / 2} H^{n+1} T_{x}\left[\begin{array}{c}
L_{x x} \\
L_{y y}
\end{array}\right],
$$

and the shock speed by

$$
v=A_{\mathrm{s}}\left(T_{x}^{2} L_{x x}\right)^{(n-1) / 2} H^{n+1} T_{x}\left(\cos \varphi L_{x x}+\sin \varphi L_{x y}\right),
$$

where $\varphi$ is the angle the shock axis makes with the $x$ axis. The dependence of the shock speed on the angle is thus different for isotropic and anisotropic beds.

\section{APPLICATIONS}

\subsection{Numerical solutions to the ice-sheet equation}

Some calculations based on the European Ice Sheet Model-

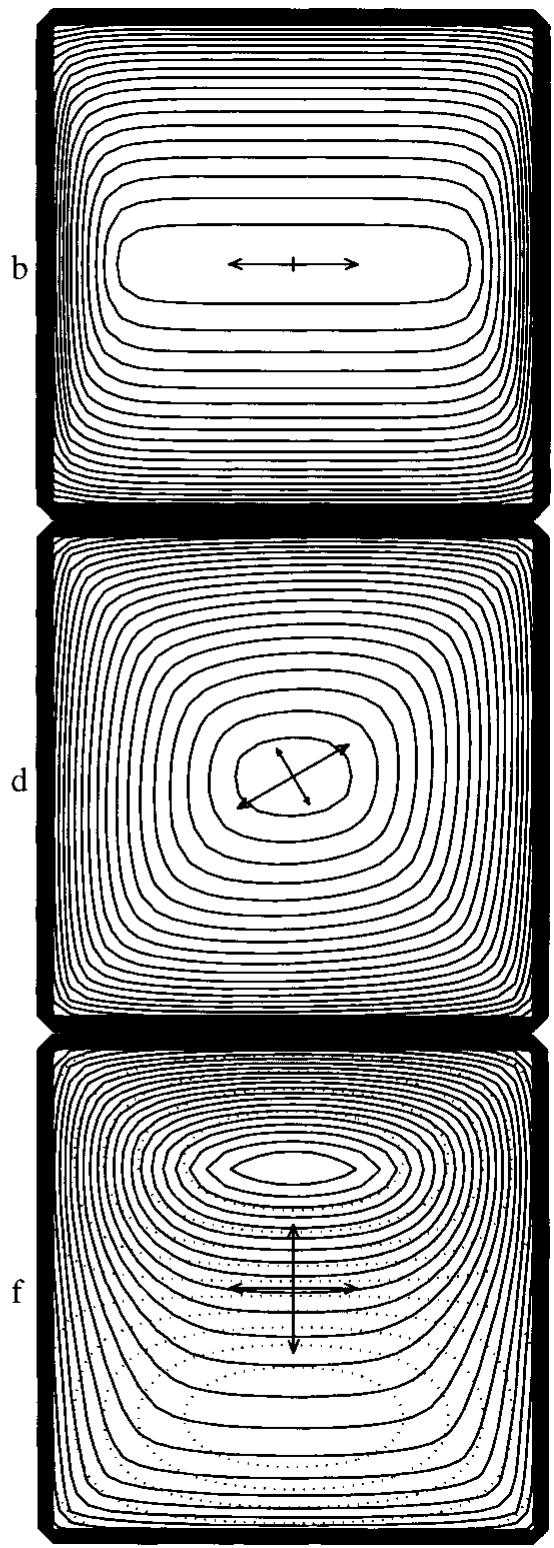

Fig. 4. The influence of bed anisotropy and bed asymmetry on steady ice-sheet profiles computed using the non-linear equations. Based on the EISMINT Benchmark configuration (Huybrechts and others, 1996). Six calculations are presented as contour plots: (a) flat bed, isotropic smoothness ( the EISMINT Benchmark); ( $b$ ) flat bed, anisotropic smoothness angled at $0^{\circ}$, principal smoothness ratio of 10:1; (c) flat bed, anisotropic smoothness angled at 30 , principal smoothness ratio of 10:1; (d) flat bed, anisotropic smoothness angled at $30^{\circ}$, principal smoothness ratio of $2: 1 ;$ (e) flat bed, anisotropic smoothness angled at $45^{\circ}$, principal smoothness ratio of 10:1; and $(f)$ asymmmetric bed, isotropic smoothness. Bed contours are shown as dotted lines; these can be seen to be asymmetrical about the centre of the solution domain. 
ling Initiative (EISMINT) Benchmark (Huybrechts and others, 1996) are presented. The basic configuration is a $1500 \mathrm{~km}^{2}$ ice sheet with zero-thickness (Vialov) boundary conditions, which admit a finite outlet flux through zero ice thickness. Accumulation is uniform. Bedrock topography is prescribed (but flat in most of the cases presented here), while flow is not through internal deformation as in the EISMINT Benchmark, but by Weertman sliding and with an anisotropic smoothness tensor using Equation (5). Six calculations are presented and the results are shown in Figure 4.

Smoothness ratios of 10:1 have a very significant effect, depending on the alignment of the principal directions with the divide ridges. This implies a wavelength ratio of around $3: 1$. Smoothness ratios of $2: 1$ have a less-marked but nevertheless observable effect. Large-scale bed asymmetry has equally marked, but qualitatively different effects.

\subsection{Rugose topography underlying cold-based ice sheets}

Cold-based ice sheets contain a shear layer at the base (Fowler, 1992) where most of the internal deformation occurs. This layer arises because the basal viscosity is lower owing to the higher stresses and temperatures. Rugose topography which substantially penetrates this layer is likely to affect the flow of ice sheets. If this topography has a grain, then cold-based ice is also likely to exhibit non-parallelism between surface slopes and flow directions. Such non-parallelism should not be used a priori to infer either warm bases or the breakdown of the shallow-ice approximation.

\section{CONGLUSIONS}

(1) Anisotropic beds give rise to non-parallel basal-tangential-traction and basal-sliding vectors, which are related by a smoothness tensor. This tensor is real and symmetric to ensure positive definiteness, necessary on account of the Second Law of Thermodynamics. This tensor can be diagonalized, with the two eigenvalues, necessarily both positive, called the principal major and principal minor smoothnesses. In the shallow-ice approximation, velocity and slope vectors are no longer parallel in general, which means that there is a distinction between upslope when considering the ice surface and upflow. Non-linear sliding requires a generalization of the absolute value of the slope in terms of a quadratic form involving the slope vector and the inverse smoothness tensor.

(2) The Nye-Kamb solution for short-wavelength obstructions predicts anisotropic sliding, with the strengths of the principal smoothnesses being proportional to the wavelength squared for linear rheologies. For wavelength ratios of $4: 1$, tractions deviating only slightly from the direction of the minor principal smoothness result in velocities strongly aligned with the major principal smoothness.

(3) For flow according to the shallow-ice approximation down the infinite plane, the decay rate of surface disturbances is given by $-Q_{0}\left(\mathbf{k}^{\mathrm{s}}\right)^{\mathrm{T}} \mathbf{S}\left[\nu_{x} k_{x}^{\mathrm{s}}, \nu_{y} k_{y}^{\mathrm{s}}\right]^{\mathrm{T}} / \varepsilon$. Plane wave velocities in the $x$ direction are given by $\left(\nu_{x}+1\right) U_{0}\left(S_{x x}+S_{x y} k_{y}^{\mathrm{s}} / k_{x}^{\mathrm{s}}\right)$. Plane waves of opposite orientation (e.g. with $k_{y}$ of opposite sign) have a different wave velocity, which means that superpositions of these plane waves, which are hummocks and hollows, have lateral velocities which depend on the relative wavelength and the orientation of the principal smoothnesses.

(4) Under steady flow, according to the shallow-ice approximation, steady anisotropic sliding can affect surfacetopography-basal-topography relationships. If the long-wavelength basal topography is of shorter wavelength in the direction of the traction vector, then the ice-surface highs are found upslope of the basal highs. If the long-wavelength basal topography is isotropic, icesurface highs reside upflow of the basal highs.

(5) Shock waves angled at $\theta$ across the direction of flow have a shock-wave velocity $\cos \theta$ of that of a wave with axis orthogonal to the flow when sliding is isotropic. Anisotropic sliding will cause there to be a different relationship between the angle subtended by the axis of a plane shock wave and the direction of maximum of slope.

(6) Anisotropic sliding causes deflection of divide ridges compared with the isotropic case. This may be of significance in East Antarctica, where basal temperatures in central regions reach melting point.

(7) Anisotropic sliding will make inferring the deposition location of snow in off-divide cores more difficult; slope strike does not equal flow strike.

\section{ACKNOWLEDGEMENTS}

Thanks to G. H. Guðmundsson and K. Hutter for their careful reviewing.

\section{REFERENCES}

Bear, J. 1972. Dynamics of fluids in porous media. New York, American Elsevier. Bindschadler, R. 1983. The importance of pressurized subglacial water in separation and sliding at the glacier bed. F. Glaciol., 29(101), 3-19.

Budd, W. F. 1970. Ice flow over bedrock perturbations. F. Glaciol., 9(55), 29-48. Fowler, A. C. 1982. Waves on glaciers. F. Fluid Mech., 120, 283-321.

Fowler, A. C. 1992. Modelling ice sheet dynamics. Geophys. Astrophys. Fluid Dyn., 63(1-4), 29-66.

Gudmundsson, G. H. 1997. Basal flow characteristics of a non-linear flow sliding frictionless over strongly undulating bedrock. F. Glaciol., 43(143), 80-89.

Hättestrand, C., D. Goodwillie and J. Kleman. 1999. Size distribution of two cross-cutting drumlin systems in northern Sweden: a measure of selective erosion and formation time length. Ann. Glaciol., 28, 146-152.

Hutter, K. 1983. Theoretical glaciology; material science of ice and the mechanics of glaciers and ice sheets. Dordrecht, etc., D. Reidel Publishing Co.; Tokyo, Terra Scientific Publishing Co.

Huybrechts, P., T. Payne and The EISMINT Intercomparison Group. 1996. The EISMINT benchmarks for testing ice-sheet models. Ann. Glaciol., 23, 1-12.

Jóhannesson, T. 1992. The landscape of temperate ice caps. (Ph.D. thesis, University of Washington.)

Kamb, B. 1970. Sliding motion of glaciers: theory and observation. Rev. Geophys. Space Phys., 8(4), 673-728.

Nye, J. F. 1959. The motion of ice sheets and glaciers. F. Glaciol., 3(26), 493-507.

Nye, J. F. 1969. A calculation on the sliding of ice over a wavy surface using a Newtonian viscous approximation. Proc. R. Soc. London, Ser. A, 311 (1506), 445-467.

Nye, J. F. 1970. Glacier sliding without cavitation in a linear viscous approximation. Proc. R. Soc. London, Ser. A, 315(1522), 381-403.

Rémy, F. and J.-F. Minster. 1997. Antarctic ice sheet curvature and its relation with ice flow and boundary conditions. Geophys. Res. Lett., 24(9), 1039-1042.

Weertman, J. 1957. On the sliding of glaciers. F. Glaciol., 3(21), 33-38. 